Apidologie, 1980, 11 (2), 105-112.

\title{
VERSUCHE ZUR BIOLOGISCHEN THERAPIE DER VARROATOSE DURCH EINE KONTROLLE DER FRISCHGESCHLÜPFTEN BIENEN
}

\author{
Essais de traitement biologique de la varroose \\ par le contrôle de toutes les abeilles fraîchement écloses
}

\author{
Nikolaus KOENIGER und Alfred SCHULZ \\ Fachbereich Biologie d.J.W.Goethe-Universität \\ Institut für Bienenkunde \\ Im Rothkopf 5, D-6370 Oberursel/Ts.
}

\begin{abstract}
SUMMARY
EXPERIMENTS ON A BIOLOGICAL TREATMENT OF VARROATOSIS BY THE CONTROL OF ALL FRESHLY EMERGED BEES
\end{abstract}

In four by Varroa infested colonies all freshly emerged bees were controlled for 48 days and only bees without Varroas were given back into their colonies. Altogether 5446 mites were found on 31333 bees. On a sheet of paper beneath the colonies 176 mites were found. At the end of the experiment all bees were washed in benzine for examination on Varroa. The result was : One colony had become free of Varroa and in the others 2,8 and 27 mites were found. These results were discussed in relation to the development of a biological treatment of Varroatosis.

\section{ZUSAMMENFASSUNG}

Bei vier von Varroa befallenen Bienenvölkern wurden 48 Tage lang alle geschlüpften Bienen auf Varroa kontrolliert und nur milbenfreie Bienen in ihre Völker zurückgesetzt. Dabei wurden auf insgesamt 31333 Bienen 5446 Milben gefunden. Auf einem Papier unter den Völkern fanden sich 176 Varroae. Am Ende des Versuchs wurde der Restbefall durch Ausschütteln der abgetöteten Bienenvölker mit Benzin festgestellt. Dabei ergab sich, dass ein Volk befallsfrei geworden war während in den anderen jeweils 2, 8 und 27 Milben nachgewiesen wurden. Diese Ergebnisse wurden in Bezug auf die Entwicklung einer biologischen Therapie der Varroatose diskutiert. 


\section{EINLEITUNG}

Varroa jacobsoni ist eine parasitäre Milbe, deren Reproduktionsphase auf der in der verdeckelten Wabenzelle befindlichen Bienenbrut abläuft. Die begatteten Varroaweibchen suchen in der Regel ältere Streckmaden auf. Nach dem Verdeckeln der Brutzelle kommt es dann auf der Nymphe bzw. Puppe zur Eilage. Dabei bleibt das Muttertier bis zum Ende der Metamorphose der Bienenpuppe in der Zelle und verlässt diese gemeinsam mit den mittlerweile fertig entwickelten Nachkommen beim Schlupf der Biene (SMIRNov 1978). Eine sichere Unterscheidung der Muttertiere von den Jungtieren ist jedoch nicht möglich.

So ist Varroa während ihrer Reproduktion einerseits durch die geschlossene Wabenzelle vor der Einwirkung acarizider Substanzen geschützt. Andererseits könnte dieses Verhalten aber auch Ansätze für die Entwicklung einer biologischen Bekämpfung bieten. Daher beschäftigt sich die hier vorgelegte Arbeit mit der Frage, wie sich eine vollständige Kontrolle der geschlüpften Bienenbrut auf die Milbenpopulation im Bienenvolk auswirkt.

\section{MATERIAL UND METHODE}

Vier Bienenvölker (7-9 besetzte Waben) aus einem Varroatose-Befallsgebiet wurden einzeln in Freilandkäfigen $(2 \times 3 \times 2 \mathrm{~m})$ untergebracht, um eine unkontrollierte Infektion von aussen zu verhindern. Die Völker befanden sich in Magazinbeuten, die durch ein Absperrgitter in zwei Abteile unterteilt waren. Unter dem Volk auf dem Boden der Beute befand sich ein mit Gaze bedecktes weisses Papier (Windel). Dort sammelten sich die im Volk gestorbenen Varroae, deren Zahl bei einer wöclentlichen Kontrolle festgestellt wurde.

Gefüttert wurde täglich mit gemahlenem Pollen und Zuckerwasser $1: 2$, dem zur Nosemaprophylaxe Fumidil B (1g/1 l) zugefügt wurde.

$\mathrm{Zu}$ Beginn des Versuchs befand sich die + vor dem Absperrgitter auf drei neuen Waben. Nach jeweils 6 Tagen wurden die mittlerweile mit Eiern und Larven besetzten Waben hinter das Absperrgitter gestellt und der + drei neue brutfreie Waben geboten. Auf diese Weise konnten (18 Tage nach Versuchsbeginn) alle sechs Tage Waben dem Volk entnommen werden, die ausschliesslich verdeckelte Brut enthielten (Alter $12-18$ Tage), und die im Brutschrank bei $34^{\circ} \mathrm{C}$ und $60-80 \%$ rel. Feuclate bis zum Schlupf aller Bienen gehalten wurden. Die Jungbienen wurden täglich entnommen und auf Milben kontrolliert. Dabei wurde die einzelne Biene sorgfältig unter einem Präpariermikroskop untersucht. Die varroafreien Bienen wurden in ihr Volk zurückgesetzt. Die befallenen Bienen wurden entweder, soweit es zeitlich möglich war, mit einem Pinsel von den aufsitzenden Milben befreit und ebenfalls zurückgesetzt oder abgetötet.

Am Ende der 72-tägigen Versuchszeit, die sich aus 18 Tagen bis zum Beginn und den 9 mal 6 Tagen für die Brutwabenentnahme ergab, wurden alle Bienen der Völker abgetötet und jeweils mit Benzin ausgeschüttelt. Mit diesem Verfahren (RITTER + RUTTNER 1980) konnte die Zahl der noch im Volk verbliebenen Milben bestimmt werden.

Es ist ohne weiteres verständlich, dass unter diesen Versuchsbedingungen (Käfighaltung) die Voraussetzungen für die Erzeugung von Brut nicht optimal waren. In Volk 4 z.B. setzte die Königin zweimal gänzlich mit der Eiablage aus und es mussten zur Fortführung des Versuches Waben mit Eiern und offener Brut aus varroafreien Völkern zugegeben werden. Die Auswirkungen dieser Brutstopps auf den Verlauf des Versuchs sind sehr deutlich aus den Ergebnissen zu crsehen (Abb. 1).

Der Versuch wurde am 7.5. begonnen und am 17.7 abgeschlossen. 


\section{ERGEBNISSE}

Die Anzahl der bei den Brutkontrollen gefundenen Milben zeigte im Verlauf der 48-tägigen Versuchszeit wesentliche Unterschiede zwischen den Versuchsvölkern.

Bei Volk Nr. 1 war die Menge der geschlüpften Brut relativ konstant (Abb. 1 a). Die Zahl der gefundenen Milben nahm von der ersten Brutentnahme bis zur siebten ab (von $754 \mathrm{zu}$ 14), und der Anstieg von der siebten (5 Milben) zur achten Periode (12 Milben) war unwesentlich. Nach 4 Perioden wurden 1483 Varroae gefunden, das waren $96,4 \%$ der insgesamt bei der Brutkontrolle aufgefundenen Milben. In der zweiten Hälfte der Versuchszeit (Periode 5-8) wurden nur noch 55 Milben entdeckt und bei der Endkontrolle (Ausschütteln aller Bienen in Benzin) konnte festgestellt werden, dass keine Varroa mehr vorhanden war (Tab. 1).

Bei Volk Nr. 4 traten dagegen grosse Schwankungen bei den pro Untersuchungsperiode geschlüpften Brutmengen auf (Abb. 1 d). So schlüpften in der dritten und sechsten Periode keine Bienen und in der vierten Periode nur 64. Bedingt durch diese Schwankungen verlief die Anzahl der pro Wabenentnahme gefundenen Milben nicht einheitlich abnehmend wie bei Volk Nr. 1. Nach 24 Tagen (4 Untersuchungsperioden) waren erst 1590 Varroae ( $74 \%$ ) gefunden und der Befund in den Perioden 5-8 war mit 558 Milben noch hoch. Bei der Endkontrolle wurden noch 27 Milben nachgewiesen (Tab. 1).

Die Völker Nr. 2 und Nr. 3 lagen bezüglich der Bruterzeugung zwischen den o.a. Befunden und bestätigten die Abhängigkeit der gefundenen Milben von der Anzahl der geschlüpften Bienen (Abb. 1 b, 1 c). Auch diese beiden Völker waren am Ende der Versuchszeit nicht befallsfrei : Volk Nr. 2 wies noch zwei Milben auf und Volk Nr. 3 acht Milben*.

Bei der Untersuchung der Stockwindeln fanden sich bei Volk Nr. 2 zwei Varroae und bei Nr. 3 vier Milben (Tab. 1). In Volk Nr. 1 und Nr. 4 starben wesentlich mehr Milben : nämlich 77 bzw. 93 Milben. Über $90 \%$ dieser Varroae wurde in der ersten Hälfte der Versuchszeit gefunden.

\section{DISKUSSION}

Zur Abschätzung der Zahl, der zu Beginn der Brutkontrolle in den Völkern befindlichen Varroae ist die Kenntnis der Reproduktionsrate der Varroa notwendig. Bei Versuchen unter vergleichbaren Bedingungen mit markierten Milben wurde gefunden, dass neben dem alten Varroaweibchen (Mutter) durchschnittlich 2,8 Jungweibchen

* Durch eine im Anschluss an den hier geschilderten Versuch durchgeführte einmalige Behandlung mit Brompropylat konnten die in den Völkern 2 und 3 noch verbliebenen 10 Varroa-Milben entfernt werden. Damit wurden also im Endergebnis durch eine kombinierte Behandlung drei von vier Völkern restlos von Varroa-Milben befreit - trotz eines recht hohen Ausgangsbefalls. 
$=$
5
5
5
0
0
0
0
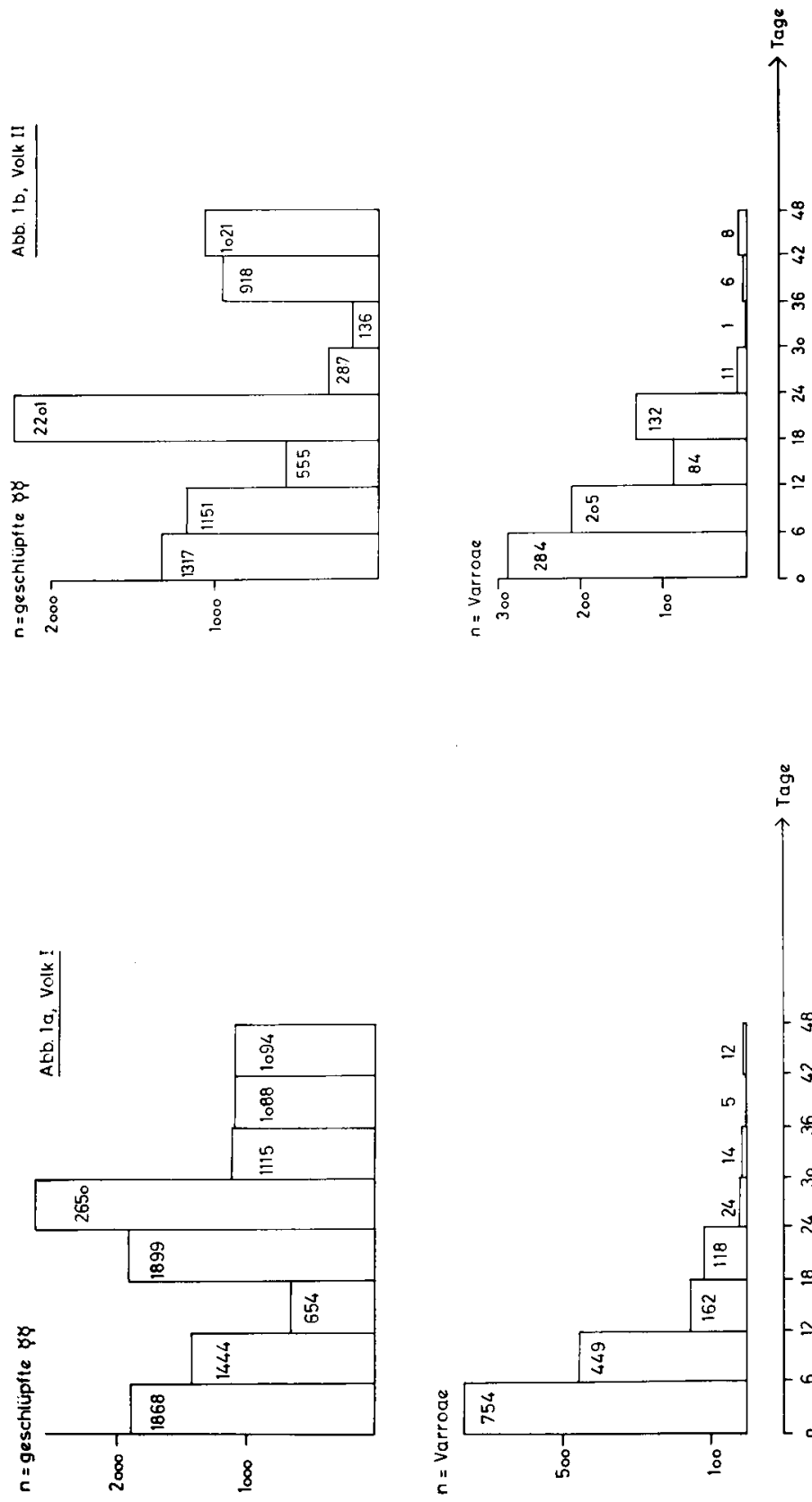

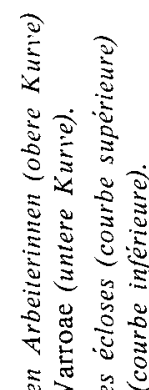

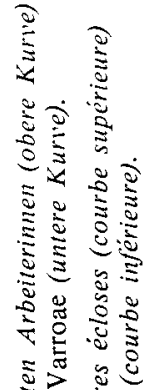

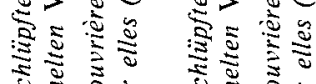

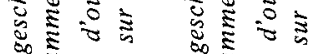

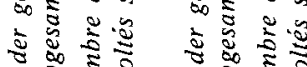

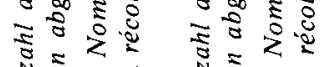

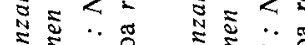

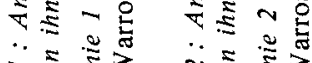

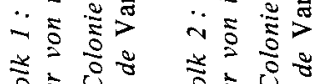

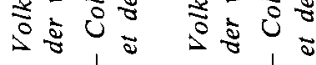

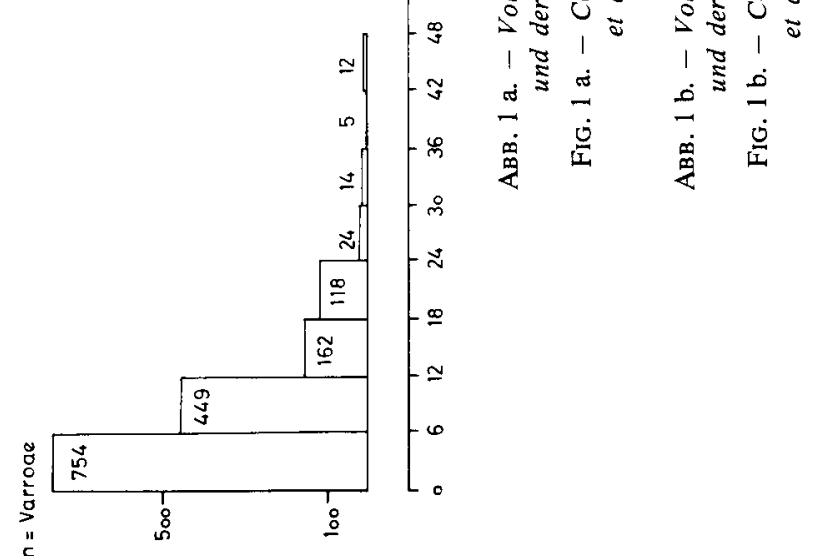




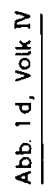
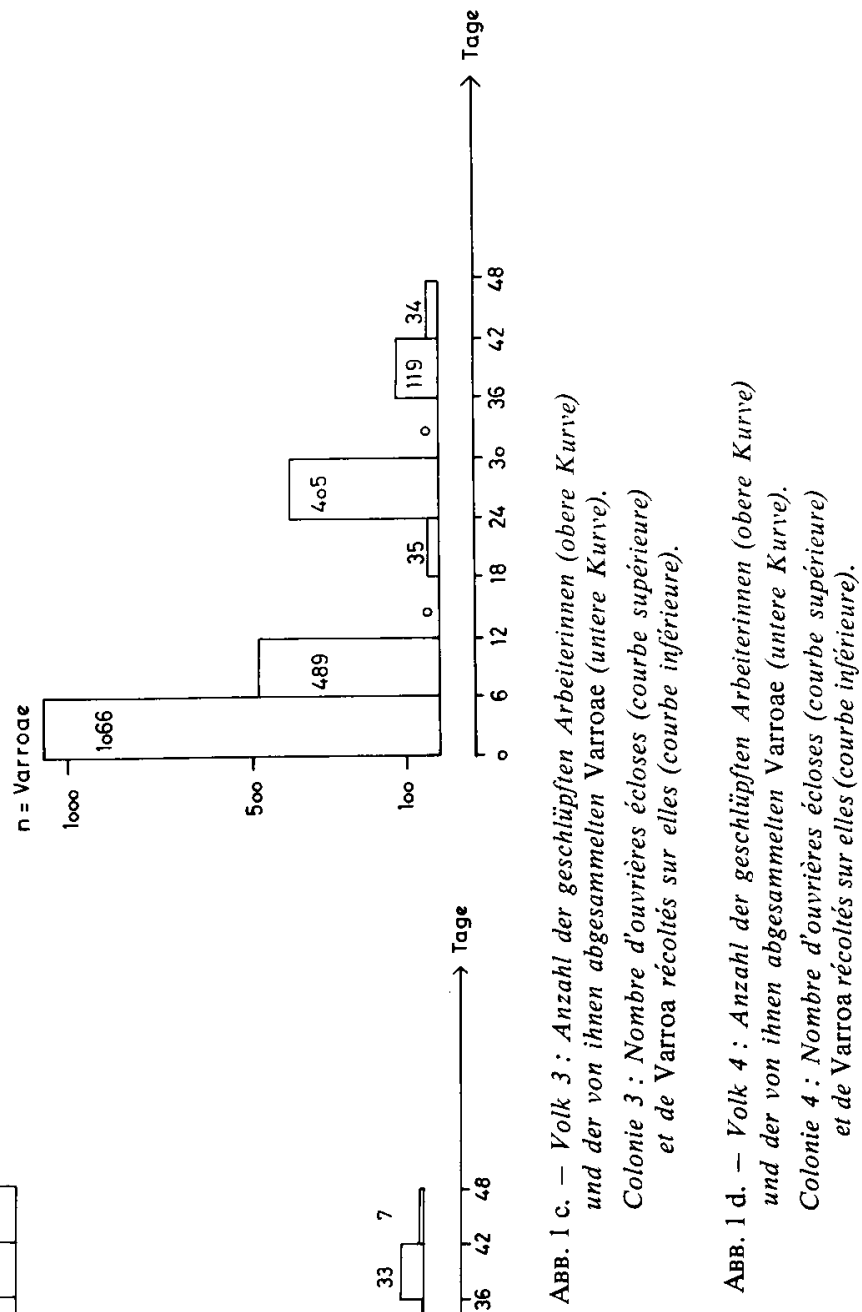

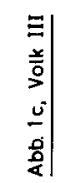
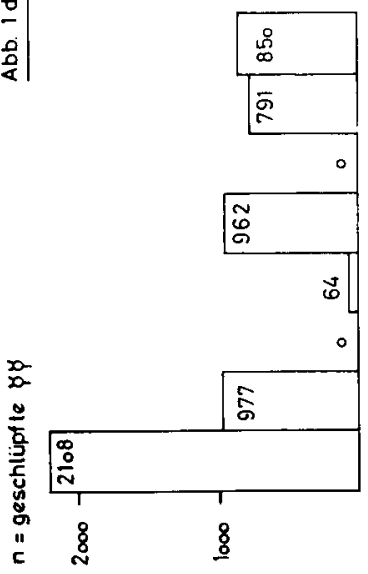

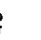


TAB. 1. - Übersicht der mit den angewandten Methoden gefundenen Varroae.

TABL. 1. - Répartition d'ensemble des Varroa trourés par les diverses méthodes utilisées.

\begin{tabular}{|c|c|c|c|c|c|}
\hline $\begin{array}{l}\text { Volk } \\
\text { Colonie }\end{array}$ & No. 1 & No. 2 & No. 3 & No. 4 & y \\
\hline $\begin{array}{l}\text { Brutkontrolle } \\
\text { Après contrôle du couvain }\end{array}$ & 1538 & 731 & 1029 & 2148 & 5446 \\
\hline $\begin{array}{c}\text { Windeln } \\
\text { Recueillis sur le papier }\end{array}$ & 77 & 2 & 4 & 93 & 176 \\
\hline $\begin{array}{l}\text { Ausschütteln } \\
\text { Après secouage dans l'essence }\end{array}$ & 0 & 2 & 8 & 27 & 37 \\
\hline$\Sigma$ & 1615 & 735 & 1041 & 2268 & 5659 \\
\hline
\end{tabular}

(Töchter) die Wabenzelle beim Schlupf der Biene verlassen (KoENIGER in Vorbereitung). Daher muss also die Zahl der bei der Brutkontrolle gefundenen Milben durch 3,8 dividiert werden, um die Zahl der Milben zu erhalten, die ursprünglich in die Brutzellen eingewandert sind. Die Ergebnisse dieser Schätzung sind in Tab. 2 dargestellt. Sie zeigen, dass von den bei allen vier Völkern vorhandenen 1646 Varroae 1433 durch die Kontrolle der geschlüpften Bienen eliminiert wurden.

Insgesamt wurden dazu 31333 Brutzellen benötigt. Daraus ergibt sich eine Zahl von 21,9 Brutzellen pro eliminierter Varroa. In diesem Durchschnittswert sind auch die Varroazahlen gegen Ende des Versuchs enthalten, als nur noch sehr wenige Milben in den Völkern vorhanden waren. Daher scheint für die Frage, wie viele Brutzellen für die Elimination einer Varroa wirklich notwendig sind, die Betrachtung der einzelnen Ergebnisse der Brutuntersuchungen (Abb. 1) geigneter zu sein. Der niedrigste Wert, 7, 1 Brutzellen/Varroa findet sich bei Volk Nr. 4 (Abb. 1 d), 19.-24. Tag. In der davor liegenden Periode war keine Brut erzeugt worden und in den folgenden sechs Tagen (25.-30.) wurden 107 Varroae entfernt, das ist eine Steigerung um einen Faktor von ca.

TAB. 2. - Anzahl der Varroae b. Versuchsbeginn

(Reproduktionsrate : 2,8 $9.9 /$ Varroamutter).

TABL. 2. - Nombre de Varroa au début de l'expérience

(Taux de reproduction : $2,8 \subsetneq q$ femelle de Varroa).

\begin{tabular}{c|c|c|c|c|c}
\hline \hline $\begin{array}{c}\text { Volk } \\
\text { Colonie }\end{array}$ & No. 1 & No. 2 & No. 3 & No. 4 & $\Sigma$ \\
\hline $\begin{array}{c}\text { Brutkontrolle } \\
\text { Après contrôle du couvain } \\
\text { Windeln } \\
\begin{array}{c}\text { Recueillis sur le papier } \\
\text { Ausschütteln }\end{array}\end{array}$ & 405 & 192 & 271 & 565 & 1433 \\
$\begin{array}{c}\text { Après secouage dans l'essence } \\
\Sigma\end{array}$ & 0 & 2 & 4 & 93 & 176 \\
\hline \hline
\end{tabular}


10. So kann vermutet werden, dass bei 7,1 Brutzellen/Varroa nicht alle reproduzierwilligen Milben eine Zelle fanden sondern bis zur nächsten Periode warten mussten.

Bei Volk Nr. 1 (Abb. 1 a) ergibt sich für die 1. Periode ein Wert von 9,4 Zellen/Varroa. Da in der folgenden Zeit niedrigere Milbenzahlen auftreten, ist dieser höhere Wert wohl ausreichend. Damit liegt das Optimum, bei dem mit Hilfe einer möglichst kleinen Anzahl Brutzellen alle fortpflanzungswilligen Milben gefangen werden, wohl zwischen 7,1 und 9,4 Brutzellen/Milbe. Die Höhe dieses Wertes ist bemerkenswert, zumal sich bei der Untersuchung von verdeckelten Zellen herausgestellt hat, dass auch zwei und mehr Varroae (reproduzierende Weibchen) in einer einzigen Zelle gefunden werden können (Groвov 1976).

Vielleicht hängt die Frage, warum ca. acht Brutzellen für den Fang einer Varroa notwendig sind, mit dem bisher völlig unbekannten Orientierungsmechanismus zusammen, der der Varroa das Finden einer für die Reproduktion geeigneten Brutzelle ermöglicht.

Insgesamt 176 Milben wurden tot auf den Stockwindeln gefunden. Es kann vermutet werden, dass in diese letztere Gruppe insbesondere die Milben fallen, die bereits die Reproduktion hinter sich haben und deren Lebensdauer danach natürlicherweise nur noch kurz ist. Für diese Erklärung spricht, dass mit fortschreitender Versuchsdauer bzw. mit zunehmendem zeitlichen Abstand von der letzten im Volk geschlüpften Brut die Milbenfunde auf den Stockwindeln abnahmen. Für die Beurteilung des Effektes der Kontrolle der schlüpfenden Brut auf den VarroaBefall scheinen daher diese Milben wenig relevant zu sein.

Wichtig in dieser Hinsicht sind die Ergebnisse des Ausschüttelns. Sie zeigen, dass Volk Nr. 1 am Ende des Versuchs keine einzige Milbe mehr enthielt und dass die Überlebensrate der Varroae bei den anderen Völkern nur zwischen $1-3 \%$ beträgt. Das ist ein wichtiger Ansatzpunkt für die Entwicklung von biologischen Bekämpfungsmethoden der Varroatose. Dabei ist eine Änderung der hier beschriebenen experimentellen Vorgehensweise in Richtung auf eine praxisorientierte, grossflächig durchführbare Behandlung der Bienenvölker notwendig. Wie erste Vorversuche zeigen, scheint eine Kombination von biologischen Verfahren wie z. B. Entfernung von vedeckelter Brut und einer anschliessenden Chemotherapie vielversprechend zu sein (RUTTNER + KOENIGER, 1979).

\section{RÉSUMÉ}

L'acarien parasite Varroa se multiplie dans les cellules operculées sur le couvain d'abeilles. Là, d'une part il est protégé contre l'action des substances chimiques acaricides, mais d'autre part ce comportement serait susceptible d'offrir des points de départ pour un traitement biologique de la varroose. 
Matériel et méthodes.

Quatre colonies d'abeilles ont été maintenues chacune dans une cage $(2 \times 3 \times 2 \mathrm{~m})$ à l'extérieur. Un grillage divisait les ruches en 2 parties. Dans la première se trouvait la reine qui, tous les 6 jours, était installée sur un rayon frais. Les rayons de couvain étaient ensuite conservés dans la seconde partie de la ruche pendant 12 jours, puis transférés dans une étuve $\left(34^{\circ} \mathrm{C}, 70 \%\right.$ d'humidité relative). On contrôlait chaque jour la présence ou l'absence d'acariens sur les abeilles qui venaient d'éclore et seules celles indemnes de Varroa étaient replacées dans leur colonie d'origine. En plus de ce contrôle, on pratiquait 2 autres méthodes :

1. Sur le sol sous la colonie on plaçait un papier sur lequel s'accumulaient les Varroa morts dans la ruche.

2. A la fin de la période d'expérimentation de 72 jours, chaque colonie a èté tuée et toutes les abeilles mortes secouées dans de l'essence. Par cette méthode (RITTER et RuTTNER, 1980) on a pu dénombrer avec exactitude les acariens encore présents dans la colonie.

Résultats et discussion.

Le nombre d'acariens trouvés lors des contrôles du couvain dépendait du nombre d'abeilles écloses (Fig. 1). On a réussi à réduire considérablement l'infestation par les acariens (Tabl. 1). Pour évaluer l'infestation de Varroa au début des contrôles de couvain, il est nécessaire de connaitre le taux de reproduction de Varroa : il est de 2,8 (KoENiger en préparation). L'infestation au début de l'expérience est représentée dans le Tabl. 2. Une vérification des résultats obtenus lors des contrôles du couvain prouve qu'il faut entre 7,1 et 9,4 cellules de couvain disponibles par acarien pour que tous les acariens capables de se reproduire puissent être éliminés.

Comme le montrent les résultats, il n'y a plus un seul acarien dans la colonie 1 à la fin de l'expérience. Dans les autres colonies le taux de survie n'est que de 1-3\%. Ceci est un point de départ important pour développer des méthodes biologiques de traitement de la varroose.

\section{LITERATUR}

Grobov O. F., 1976. - Varroasis in bees. - IOE/Apimondia-Symposium "Varroasis a honeybee disease ", Sofia, Bulgaria p. 46-90.

Koeniger N., 1980. - Beobachtungen an markierten Milben (Varroa jacobsoni). In Vorbereitung.

Ritter W., RutTner F., 1980. - Versuche zur Chemotherapie der Varroatose der Honigbiene. Apidologie im Druck.

RutTNER F., Koeniger N., 1979. - Eine biologische Methode zur Eliminierung der Varroa-Milben aus Bienenvölkern. XXVII. Internat. Bienenzüchterkongress Apimondia, Athen, Im Druck.

SmiRnov A. M., 1978. - Ätiologie, Diagnose und Bekämpfung der Varroatose. OIE/Apimondia-Seminar "Bekämpfung und Vorbeugung der Varroatose ", Bukarest, Rumänien S. 60-74. 